\title{
Detecting Land Cover Change Using an Extended Kalman Filter on MODIS NDVI Time Series Data
}

\author{
W. Kleynhans, J.C. Olivier, K.J. Wessels, B.P. Salmon, F. van den Bergh and K. Steenkamp
}

\begin{abstract}
A method for detecting land cover change using NDVI time series data derived from $500 \mathrm{~m}$ MODIS satellite data is proposed. The algorithm acts as a per pixel change alarm and takes as input the NDVI time series of a $3 \times 3$ grid of MODIS pixels. The NDVI time series for each of these pixels was modeled as a triply (mean, phase and amplitude) modulated cosine function, and an Extended Kalman Filter was used to estimate the parameters of the modulated cosine function through time. A spatial comparison between the center pixel of the the 3x3 grid and each of its neighboring pixel's mean and amplitude parameter sequence was done to calculate a change metric which yields a change or no-change decision after thresholding. Although the development of new settlements is the most prevalent form of land cover change in South Africa, it is rarely mapped and known examples amounts to a limited number of changed MODIS pixels. Therefore simulated change data was generated and used for preliminary optimization of the change detection method. After optimization the method was evaluated on examples of known land cover change in the study area and experimental results indicate a $89 \%$ change detection accuracy, while a traditional annual NDVI differencing method could only achieve a $63 \%$ change detection accuracy.
\end{abstract}

Index Terms - change detection, extended Kalman filter, timeseries

\section{INTRODUCTION}

Anthropogenic land cover change has a major impact on hydrology, climate and ecology [1]. Remote sensing satellite data provide researchers with an effective way to monitor and evaluate land cover changes [2], [3]. Operator-dependent image to image comparison is still the most prevalent way of operationally mapping change, but is time consuming and resource intensive. Automated change detection reduces human interaction and enables large datasets to potentially be processed in a fraction of the time. Using multi-temporal coarse resolution satellite imagery to perform automated land cover change detection has however proved challenging [4]. Change detection using coarse resolution data could potentially be used as a first step in alerting and tasking high resolution satellites of potential change events within the envisaged autonomous Earth Observation sensor web [5]. Fully supervised change detection methods using temporal satellite data have shown potential, but require a considerable amount of training data to be useful [6], [7], [8], [4]. The dearth of regional land cover training data makes unsupervised change detection a more attractive solution.

W. Kleynhans and B.P. Salmon is with the Department of Electrical, Electronic and Computer Engineering, University of Pretoria as well as the Remote Sensing Research Unit, Meraka Institute, CSIR, Pretoria, South Africa. Email: wkleynhans@csir.co.za. Telephone: +27 84807 2419. Fax: +27 128413124 .

J.C. Olivier is with the Department of Electrical, Electronic and Computer Engineering, University of Pretoria as well as Defense, Peace, Safety and Security, CSIR, Pretoria, South Africa

K.J. Wessels, F. van den Bergh and K. Steenkamp is with the Remote Sensing Research Unit, Meraka Institute, CSIR, Pretoria, South Africa
Unsupervised methods make use of a change metric that indicates the level of change. In many cases, Univariate Image Differencing (UID) is used [9]. Two spatially registered high resolution images acquired at two different instances are subtracted on a pixel basis. Each pixel is classified as either belonging to the change or no change class by comparing the difference of two co-located pixels to a threshold value [10],[11]. The selection of this threshold value is however not a trivial task [12], [13]. The problem with the comparison of only two images is that similar land cover types can appear significantly different at various stages of the natural growth seasonal cycle [14]. The temporal frequency of the remote sensing data acquisitions should thus be adequate to distinguish change events from phenological cycles [15], [16]. The high temporal frequency of course spatial resolution imagery makes it very attractive for change detection [17]. Recently, a method proposed in [14] explored the use of multi-temporal Moderate-resolution Imaging Spectroradiometer (MODIS) Normalized Difference Vegetation Index (NDVI) data to provide an automated change detection alarm.

It was proposed in [18] that the MODIS 8-day NDVI time series be modeled as a single, but triply modulated cosine function, where the mean $\mu$, amplitude $\alpha$ and the phase $\phi$ values are a function of time. The parameters of the triply modulated cosine function were estimated using a non-linear extended Kalman filter (EKF) and the consequent EKF derived parameter sequences of the mean and amplitude were found to be highly separable for natural vegetation and settlement land cover types [18]. This paper extended this methodology to the change detection case by calculating a change metric by means of spatial comparison of the EKF derived mean and amplitude parameter sequences of any given pixel with that of its neighboring pixels (hereafter referred to as the EKF change detection method). The underlying idea is that if the center pixel's parameter sequences change significantly relative to the parameter sequences of its neighboring pixels, a change is detected. It should also be noted that a change can be detected in the event that the neighboring pixels change concurrently with the center pixel, as this influences the neighborhood statistics.

The objective was to demonstrate that by making use of the spatial, EKF-derived change metric and a threshold optimized using simulated land cover change, an unsupervised change detection method can be formulated that accurately detects change using MODIS NDVI time series data. In particular, the approach was used to detect new settlement developments in a timely and effective manner, being able to detect change affecting but a few MODIS pixels.

Making use of simulated or synthetic data is a well known concept in the remote sensing community [12], [19], [20]. In this paper, the use of simulated change data is twofold. Firstly, during development of the new method the simulated change data was used to optimize the method and to tentatively evaluate the performance of the algorithm. Simulation was 


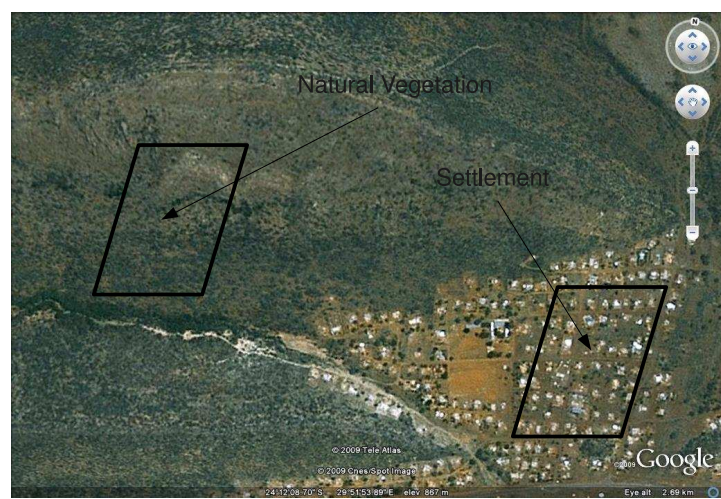

Fig. 1. 500m MODIS Pixel covering Natural Vegetation and Settlement land cover in close proximity (courtesy of Google ${ }^{\mathrm{TM}}$ Earth)

opted for during the optimization phase, since new settlements are infrequently mapped on an ad hoc basis in South Africa and the data on known settlement development amount to a relatively small number of MODIS pixels. Second, the start date and rate of the land cover change could be controlled in the simulated or synthetic data which greatly facilitates the development and evaluation phases. After the method was optimized and performing well on simulated (synthetic) change data, it was evaluated by applying it to examples of known new settlement development in South Africa. The change detection accuracy of the EKF method was also compared with that of an annual MODIS NDVI differencing method introduced by Lunetta et al. [14].

\section{DATA DESCRIPTION}

\section{A. Study Area}

The study area is located in northern South Africa and is mostly covered by natural vegetation which predominantly consist of grassland, savanna and shrubland. A large number of informal settlements are however rapidly expanding throughout the area. The study area covers an approximate $25000 \mathrm{~km}^{2}$ having an upper left coordinate of $\left(23^{\circ} 20^{\prime} 12.09^{\prime \prime} \mathrm{S} ; 28^{\circ} 35^{\prime} 25.18^{\prime \prime} \mathrm{E}\right)$ and a lower right coordinate of $\left(25^{\circ} 00^{\prime} 14.59^{\prime \prime} \mathrm{S} ; 30^{\circ} 06^{\prime} 58.30^{\prime \prime} \mathrm{E}\right)$.

A total of 1497 examples of natural vegetation $500 \mathrm{~m}$ MODIS pixels and 1735 examples of settlement 500m MODIS pixels were identified within the study area. Each of these pixels were evaluated using SPOT5 high resolution data to ensure that none of them have experienced any land cover change during the study period.

Of the available 1479 natural vegetation and 1735 settlement pixels identified in the study area, a subset of 750 natural vegetation and 750 settlement pixels were used in the development phase to create a simulated change and no-change dataset used to tentatively evaluate the change detection method. The remaining pixels where used together with the examples of real change to determine the accuracy of the change detection method.

\section{B. MODIS Data}

The $250 \mathrm{~m}$ MODIS NDVI (MOD13) data was initially considered for this application, but the bidirectional reflectance distribution function (BRDF) effects caused a high degree of noise. The NDVI time series data was therefore derived from 8 daily composite MCD43 BRDF-corrected, MODIS data with a spatial resolution of $500 \mathrm{~m}$ [21] for the period

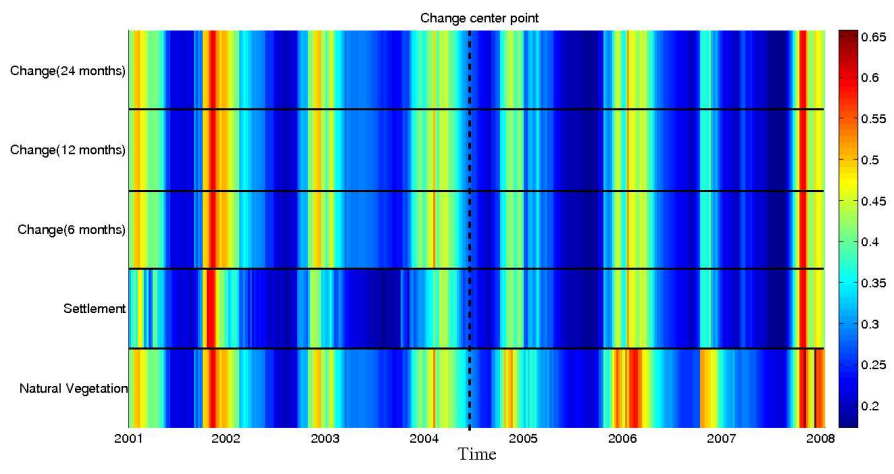

Fig. 2. NDVI time series of natural vegetation, settlement and simulated change pixels where the simulated change time series had blending periods of 6,12 and 24 months respectively.

2001/01 to 2008/01. As the majority of traditional change detection methods using time series data is based on NDVI [14], it was used in this paper to maximize comparability. The use of Enhanced Vegetation Index (EVI) was considered but preliminary results showed an insignificant performance gain over NDVI for the proposed method.

\section{Simulated Change Data}

Simulated change data were created by linearly blending a time series of a pixel covered by natural vegetation with that of a pixel of a settlement which is in close proximity to ensure that the rainfall, soil type and local climate was similar. Figure 1 shows a typical MODIS pixel covered by natural vegetation and a settlement pixel in close proximity. Figure 2 shows the corresponding NDVI time series from 2001/01 to 2008/01 for each of these pixels as well as the simulated time series where the blending period was set at 6,12 and 24 months respectively with the midpoint of the blending period being 2004/04. The exact date of change does not affect the method as long as the change does not occur within the first two years of the NDVI time series. The reason for this is that an initial two year period should be allowed for the EKF to effectively track the parameters of the triply modulated cosine model for each NDVI time series [18]. As will be described in section III, the algorithm uses a $3 \times 3$ pixel grid with the center pixel being compared to all neighboring pixels. It is however not realistic to assume that only the center pixel has changed with all neighboring pixels remaining unchanged. For this reason, the center pixel together with a range of neighboring pixels (zero to all eight) were subjected to a simulated land cover change. The simulated change for each of the neighboring pixels was done in a similar manner ensuring that the initial state of each neighboring pixel is in a vegetated state and gradually blends to a settlement state.

\section{Known Change Data}

Examples of confirmed settlement development were obtained by means of visual interpretation of high resolution Landsat and SPOT images in 2000 and 2008 respectively. All settlements identified in 2008 were referenced back to the same physical area in 2000 and all the new settlement polygons were mapped and the corresponding MODIS pixels $(n=117)$ were so identified. At least $70 \%$ of the pixel had to have changed for inclusion into the real change dataset. The data on known settlement development were used to obtain experimental results using the EKF change detection method developed and optimized with the aid of the simulated data set. 


\section{Methodology}

\section{A. EKF framework}

The NDVI time series for a given pixel was modeled by a triply modulated cosine function given as

$$
y_{k}=\mu_{k}+\alpha_{k} \cos \left(\omega k+\phi_{k}\right)+v_{k},
$$

where $y_{k}$ denotes the observed value of the NDVI timeseries at time $k$ and $v_{k}$ is the noise sample at time $k$. The cosine function is based on the angular frequency $\omega$, mean $\mu$, amplitude $\alpha$ and the phase $\phi$. The angular frequency can be explicitly computed as $\omega=2 \pi f$ where $f$ is based on the annual vegetation growth cycle. Given the 8 daily composite MCD43 MODIS data, $f$ was calculated to be $8 / 365$. The values of $\mu_{k}, \alpha_{k}$ and $\phi_{k}$ are functions of time, and must be estimated given $y_{k}$ for $k \in 1, \ldots, N$. An EKF was used to estimate these parameters for every increment of $k$. The estimated values for $\mathbf{x}_{k}=\left[\begin{array}{ll}\mu_{k} & \alpha_{k} \phi_{k}\end{array}\right]^{T}$ over time $k$ effectively results in a time series for each of the three parameters [18].

\section{B. EKF Change Detection Method}

Having the parameter sequence for $\mu_{k}, \alpha_{k}$ and $\phi_{k}$ for $k \in$ $1, \ldots, N$ for a given pixel, a change detection method was formulated by comparing the parameter sequences of the pixel with that of its direct neighboring pixels. This effectively means focusing on the center pixel of a $3 \times 3$ grid of pixels and examining each neighboring pixel's EKF parameter sequence relative to the center pixel. It was previously established that the $\phi$ parameter sequence does not yield any significant separability between natural vegetation and settlement land cover types and consequently only the $\mu$ and $\alpha$ parameter sequences were considered [18]. The $\mu$ and $\alpha$ parameter sequence difference between the center pixel and an arbitrary neighboring pixel at time $k$ can be written as

$$
\begin{aligned}
& D_{\mu(n)}^{k}=\left|\mu_{k}-\mu_{k}^{n}\right| \quad n \in 1, \ldots, 8, \\
& D_{\alpha(n)}^{k}=\left|\alpha_{k}-\alpha_{k}^{n}\right| \quad n \in 1, \ldots, 8,
\end{aligned}
$$

where $D_{\mu(n)}^{k}$ is the distance between the $\mu$ parameter sequence of a selected pixel $\left(\mu_{k}\right)$ with its $n$ 'th neighboring pixel $\left(\mu_{k}^{n}\right)$ at time $k . D_{\alpha(n)}^{k}$ is the distance between the $\alpha$ parameter sequence of a selected pixel $\left(\alpha_{k}\right)$ with its $n$ 'th neighboring pixel $\left(\alpha_{k}^{n}\right)$ at time $k$. Equation 2 and 3 can be combined as

$$
D_{n}^{k}=D_{\mu(n)}^{k}+D_{\alpha(n)}^{k} n \in 1, \ldots, 8 .
$$

Having obtained the distance of the center pixel's parameter sequences relative to each of the neighboring pixel's parameter sequences, these could be combined at time $k$ by simply adding all the values of $D_{n}^{k} n \in 1, \ldots, 8$ at time $k$

$$
D^{k}=\sum_{n=1}^{8} D_{n}^{k} \quad k \in 1, \ldots, N .
$$

Having vector $\mathbf{D}=\left[\begin{array}{llll}D^{1} D^{2} D^{3} \ldots D^{N}\end{array}\right]$, a change metric was derived by firstly determining how the relative distance of the parameter sequences between the center pixel and its neighboring pixel changes through time. This was done by differentiating the vector $D$. A single change metric was then derived by summing all the values of the differentiated $D$ vector to yield

$$
\delta=\sum_{k=2}^{N}\left|D^{k}-D^{k-1}\right|
$$

where $\delta$ is a single valued change metric for the center pixel of the $3 \times 3$ pixel grid. The change metric for each of the pixels in the study area was thus calculated by sliding a $3 \times 3$ pixel grid over the entire study area and calculating $\delta$ for the center pixel in each case.

\section{Annual NDVI Differencing Method}

The EKF change detection method was compared to a computationally simple change detection method proposed by Lunetta et al. [14]. Using this method, the NDVI time series was firstly filtered and cleaned using Fourier transformation filtering, thereafter the cumulative annual NDVI for each of the pixels in the study area were calculated and differenced for consecutive years. A normal distribution is estimated for the difference values of each year. Using standard normal distribution statistics, the pixels exhibiting the largest reduction in total annual NDVI are labeled as changed pixels [14].

\section{RESUlTS AND Discussion}

\section{A. Optimizing the Method Using Simulated Change}

The algorithm was firstly run on examples of natural vegetation pixels known not to have changed within the seven year study period. The value of $\delta$ as calculated in Equation 6 was recorded for each pixel. Next, the algorithm was run on the simulated changed pixels as described in section II-C where the blending period was 6,12 and 24 months respectively. The value of $\delta$ was again calculated and recorded for each pixel.

The change detection accuracy of the change detection method having a varying blending period (6,12 and 24 months) as well as varying number of pixels having changed within the $3 \times 3$ pixel grid is shown in table I. The true positive (TP) and true negative (TN) values, which corresponds to the change detection accuracy and false alarm rate respectively, together with the optimal decision threshold $\left(\delta^{*}\right)$ is shown for each instance. The optimal threshold was calculated by considering the probability density function of $\delta$ in the case of change and no-change respectively and calculating the decision boundary that minimized the total probability of error. It is evident from the results shown in table I that the overall accuracy of the algorithm decreases as the number of changed pixels within the $3 \times 3$ grid increases. This is to be expected as the spectral signatures of settlement pixels in close proximity (i.e. the final state of all the changed pixels in the $3 \times 3$ grid), are very similar. The pixels subjected to change within the $3 \times 3$ pixel grid would thus be correlated. This implies that the average distance between the center and neighboring pixel's parameter stream would decline, which effectively reduces separability of the distribution of $\delta$ for the change and no-change case respectively. These results using the simulated change data provides an optimization method for establishing threshold values of the change metric. The ability of the proposed method to detect real change was tested next.

\section{B. Change Detection Accuracy for Known Change}

As with most unsupervised change detection methods, the selection of a suitable threshold is a non-trivial task [10]. As was shown in the simulated change experiment results (table I), the optimal threshold $\delta^{*}$ varied between depending on the rate of change as well as the number of pixels changing in the $3 \times 3$ pixel grid. By lowering the threshold, the change detection rate increases at the cost of increasing the number of false alarms. The approach in selecting the threshold for real change detection was to firstly determine the range of the threshold by 
TABLE I

True Positive (TP), False Positive (FP) AND optimal threshold $\delta *$ FOR EACH BLEND PERIOD AND NUMBER OF PIXELS HAVING CHANGED

\begin{tabular}{|c|c|c|c|}
\hline $\begin{array}{c}\text { \# of pixels } \\
\text { changed in } \\
\mathbf{3 x 3} \text { grid }\end{array}$ & $\begin{array}{c}\mathbf{6} \text { month } \\
\text { blend }\end{array}$ & $\begin{array}{c}\mathbf{1 2} \text { month } \\
\text { blend }\end{array}$ & $\begin{array}{c}\mathbf{2 4} \text { month } \\
\text { blend }\end{array}$ \\
\hline \multirow{3}{*}{1} & $\mathrm{TP}=92 \%$ & $\mathrm{TP}=92 \%$ & $\mathrm{TP}=100 \%$ \\
& $\mathrm{FP}=8 \%$ & $\mathrm{FP}=8 \%$ & $\mathrm{FP}=13 \%$ \\
$\delta^{*}=1.68$ & $\delta^{*}=1.68$ & $\delta^{*}=1.51$ \\
\hline \multirow{3}{*}{2} & $\mathrm{TP}=92 \%$ & $\mathrm{TP}=92 \%$ & $\mathrm{TP}=100 \%$ \\
& $\mathrm{FP}=8 \%$ & $\mathrm{FP}=8 \%$ & $\mathrm{FP}=13 \%$ \\
& $\delta^{*}=1.66$ & $\delta^{*}=1.66$ & $\delta^{*}=1.51$ \\
\hline \multirow{3}{*}{3} & $\mathrm{TP}=92 \%$ & $\mathrm{TP}=93 \%$ & $\mathrm{TP}=100 \%$ \\
& $\mathrm{FP}=8 \%$ & $\mathrm{FP}=9 \%$ & $\mathrm{FP}=13 \%$ \\
& $\delta^{*}=1.65$ & $\delta^{*}=1.64$ & $\delta^{*}=1.51$ \\
\hline \multirow{3}{*}{5} & $\mathrm{TP}=92 \%$ & $\mathrm{TP}=92 \%$ & $\mathrm{TP}=100 \%$ \\
& $\mathrm{FP}=9 \%$ & $\mathrm{FP}=10 \%$ & $\mathrm{FP}=13 \%$ \\
& $\delta^{*}=1.63$ & $\delta^{*}=1.62$ & $\delta^{*}=1.51$ \\
\hline \multirow{3}{*}{6} & $\mathrm{TP}=93 \%$ & $\mathrm{TP}=93 \%$ & $\mathrm{TP}=88 \%$ \\
& $\mathrm{FP}=10 \%$ & $\mathrm{FP}=11 \%$ & $\mathrm{FP}=16 \%$ \\
& $\delta^{*}=1.6$ & $\delta^{*}=1.58$ & $\delta^{*}=1.44$ \\
\hline \multirow{3}{*}{8} & $\mathrm{TP}=93 \%$ & $\mathrm{TP}=93 \%$ & $\mathrm{TP}=85 \%$ \\
& $\mathrm{FP}=11 \%$ & $\mathrm{FP}=12 \%$ & $\mathrm{FP}=19 \%$ \\
& $\delta^{*}=1.56$ & $\delta^{*}=1.54$ & $\delta^{*}=1.4$ \\
\hline \multirow{3}{*}{7} & $\mathrm{TP}=93 \%$ & $\mathrm{TP}=92 \%$ & $\mathrm{TP}=82 \%$ \\
& $\mathrm{FP}=13 \%$ & $\mathrm{FP}=13 \%$ & $\mathrm{FP}=23 \%$ \\
& $\delta^{*}=1.52$ & $\delta^{*}=1.51$ & $\delta^{*}=1.36$ \\
\hline & $\mathrm{TP}=92 \%$ & $\mathrm{TP}=90 \%$ & $\mathrm{TP}=72 \%$ \\
& $\mathrm{FP}=15 \%$ & $\mathrm{FP}=15 \%$ & $\mathrm{FP}=24 \%$ \\
& $\delta^{*}=1.47$ & $\delta^{*}=1.46$ & $\delta^{*}=1.34$ \\
\hline & $\mathrm{TP}=90 \%$ & $\mathrm{TP}=90 \%$ & $\mathrm{TP}=64 \%$ \\
& $\delta^{*}=1.42$ & $\delta^{*}=1.4$ & $\delta^{*}=1.36$ \\
\hline
\end{tabular}

anticipating the rate and area of change that is characteristic of the type of change that is expected. New settlement formations in the study area where found to typically (more than $90 \%$ of the time) be between 0.25 and $1 \mathrm{~km}^{2}$ which relates to between one and four $500 \mathrm{~m}$ MODIS pixels. Therefore, the $3 \times 3$ neighborhood analysis should be very effective for this type of land cover change.

The rate of real land cover change is very difficult to determine with only two satellite images (one Landsat image in 2000 and one SPOT image in 2008), and may vary from 6 months to 24 months. From simulation results shown in table I, the optimal threshold ranged between 1.68 for a one pixel change with a land cover transition of 6 months and 1.51 for a four pixel change with a land cover transition of 24 months. The best change detection rate will be achieved by selecting a lower threshold with the trade-off being a higher false alarm rate. The corresponding false alarm rates for the aforementioned threshold values are $8 \%$ and $13 \%$ respectively. The task at hand is thus to select the maximum allowable false alarm rate in this range keeping in mind that the highest false alarm rate would yield the best change detection accuracy. Unfortunately, the optimal threshold will vary in this range of values based on the number of pixels changing and duration of the change and can not be explicitly inferred (Table I). In our case, a 13\% threshold was selected as this was found to be a tolerable false alarm rate given the fact that the change detection accuracy would not be compromised.

The proposed algorithm was then deployed in the detection of new settlement formations using real change data. Figure 3 shows the $\mu$ parameter sequence for a center pixel that was

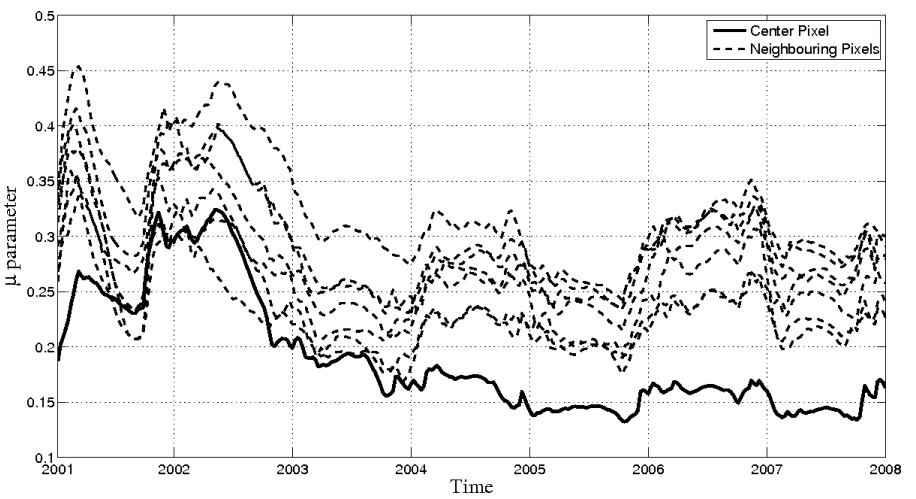

Fig. 3. Mean parameter sequence comparison of a $3 \times 3$ pixel grid with the center pixel experiencing a real change.

known to have changed. It is evident seen that as with the simulated change experiment, the real change center pixel $\mu$ parameter stream deviates from that of its neighboring pixels.

To directly compare the performance of the EKF and annual NDVI differencing change detection methods, the corresponding negative threshold ( $z$ value) was chosen so that the false alarm rate for the method in [14] would also be $13 \%$. The false alarm rate could easily be determined by considering a set of no-change pixels in the study area, the availability of which is typically not problematic given the ratio between change and no-change pixels in a regional landscape. It is evident from table II that the change detection accuracy of the annual NDVI differencing method [14] at a false alarm rate of $13 \%$ was $63 \%$ whereas the change detection accuracy of the EKF change detection method is $89 \%$ which corresponds with the change detection accuracy for the simulated change data as shown in table I.

To demonstrate that the selection of a $13 \%$ false alarm rate did not significantly disadvantage either algorithm, the performance of each algorithm at the optimal threshold was also reported in table II from which it is clear that the preselected threshold was within $10 \%$ of the optimal threshold of each algorithm. A possible explanation for the difference in the performance of the two methods could be that the EKF method considers a $3 \times 3$ grid of pixels whereas the annual NDVI differencing method uses a statistical approach that considers all the pixels in the study area. The latter approach could have difficulty in detecting land cover change when the study area is inhomogeneous (for example due to rainfall variations etc.) and the difference in annual NDVI of some no-change areas in the study area is comparable with changed pixels. The NDVI differencing method reduces the eight day composited time series over the seven year period to an effective seven observations by only considering the total annual NDVI value for each year. The EKF method, on the other hand, makes use of the entire time series by updating the mean and amplitude parameter sequence for each eight day observation and spatially comparing the parameter sequences with all the neighboring pixels. This allows for real time spatial comparison of the model parameters, which was shown in [18] to be separable for settlement and vegetation land cover types.

To demonstrate that the accuracy of the proposed EKF change detection method lies in combining the spatial approach with the EKF derived parameter sequences, an experiment was done using the spatial comparison of the NDVI time-series of the $3 \times 3$ pixel grid. The methodology proposed in section III-B was used in the exact same way, the only difference being that instead of the EKF parameter sequences being compared, the actual NDVI time-series for each pixel 
TABLE II

Real Change Detection

\begin{tabular}{|c|c|c|c|c|}
\hline Algorithm & $\begin{array}{c}\# \\
\text { Real } \\
\text { Change } \\
\text { Pixels }\end{array}$ & TP & FP & Threshold \\
\hline $\begin{array}{c}\text { Optimal Threshold } \\
\text { EKF method }\end{array}$ & 117 & $94 \%$ & $18 \%$ & $\delta^{*}=1.4$ \\
NDVI Diff. method [14] & 117 & $75 \%$ & $23 \%$ & $z^{*}=1.9$ \\
\hline $\begin{array}{c}\text { Fixed false alarm rate } \\
\text { EKF method }\end{array}$ & 117 & $89 \%$ & $13 \%$ & $\delta=1.5$ \\
NDVI Diff. method [14] & 117 & $63 \%$ & $13 \%$ & $z=2.1$ \\
\hline
\end{tabular}

was used. A change detection accuracy of $72 \%$ was obtained at a false alarm rate of $13 \%$. Although this approach did perform better than the NDVI differencing method, a further improvement was obtained by using the EKF change detection method as was shown in Table II.

\section{CONCLUSION}

In this paper, a new land cover change detection method is proposed and tested. The method models an NDVI time series as a triply modulated cosine function and estimates the mean, amplitude and phase for each time increment using an EKF. A change index was derived by comparing each pixel's mean and amplitude parameters with that of its neighboring pixels, effectively considering the center pixel of a $3 \times 3$ grid of pixels. The threshold that determined whether the change index associated with each pixel should be classified as change or no-change was determined by means of land cover change simulation. The algorithm was tested on new settlement developments in South Africa using examples of known settlement development. A change detection accuracy of $89 \%$ with a $13 \%$ false alarm rate was achieved, which was a significant improvement on computationally simple annual NDVI differencing.

Many multi date image change detection methods are not true time series analysis methods as a pixel's time series is not considered as a sampled data stream. True time series analysis is often overlooked when considering change detection methods [22]. The proposed EKF method makes full use of the NDVI time series by considering each eight day observation when updating the mean, amplitude and phase parameters of the cosine model and is inherently resilient to noise in the time series [18]. As opposed to annual NDVI differencing and image to image comparison, this allows the EKF method to give an accurate indication of when a pixel started to deviate relative to neighboring pixels (Figure 3) by comparing the center pixel's parameter sequences with that of its neighboring pixels.

The use of MODIS data for land cover change detection using various methods has been widely pursued since the inception of MODIS (e.g. [4], [17], [14], [23]). Although some MODIS change detection products do exist (such as MODIS burn-scar detection [24]), there are currently no operational MODIS change detection products tailored specifically for the change detection problem discussed in this paper. This required the development of a custom change detection method. The change detection method proposed in this paper shows promising results, as its unsupervised nature lends itself to operational viability. Even though the change detection method was evaluated for the case of detecting new settlement developments, the method shows potential to be extended to detect other forms of land cover change, and is currently under investigation.

\section{REFERENCES}

[1] R. T. Watson, Land Use, Land-Use Change and Forestry. Cambridge, England: Cambridge University Press, 2000.

[2] D. Lu, P. Mausel, E. Brondizio, and E. Moran, "Change detection techniques," International Journal of Remote Sensing, vol. 25, no. 12, pp. 2365-2407, June 2004 .

[3] P. Coppin et al., "Digital change detection methods in ecosystem monitoring: a review," International Journal of Remote Sensing, vol. 24, no. 9, pp. 1565-1596, May 2004.

[4] X. Zhan et al., "Detection of land cover changes using MODIS $250 \mathrm{~m}$ data," Remote Sensing of Environment, vol. 83, no. 1-2, p. 336350, Nov. 2002.

[5] S. Chien et al., "An autonomous Earth-Observing Sensorweb," IEEE Intelligent Systems, vol. 20, no. 3, pp. 16-24, May 2005.

[6] M. C. Hansen, R. DeFries, J. R. G. Townshend, and R. Sohlberg, "Global land cover classification at $1 \mathrm{~km}$ spatial resolution using a classification tree approach," International Journal of Remote Sensing, vol. 21, no. 6/7, pp. 1331-1364, Apr. 2000.

[7] B. H. Braswell, S. C. Hagen, S. E. Frolking, and W. A. Salas, "A multivariable approach for mapping sub-pixel land cover distributions using MISR and MODIS: Application in the Brazilian amazon region," Remote Sensing of Environment, vol. 687, no. 2-3, pp. 243-256, Oct. 2003.

[8] D. Morton et al., "Rapid assessment of annual deforestation in the Brazilian amazon using MODIS data," Earth Interactions, vol. 9, no. 8, pp. 1-22, Oct. 2005.

[9] A. Singh, "Digital change detection techniques using remotely-sensed data," International Journal of Remote Sensing, vol. 10, no. 6, pp. 9891003, June 1989.

[10] G. Moser, S. Serpico, and G. Vernazza, "Unsupervised change detection from multichannel SAR images," IEEE Geoscience and Remote Sensing Letters, vol. 4, no. 2, pp. 278-282, Apr. 2007.

[11] R. Radke, S. Andra, O. Al-Kofahi, and B. Roysam, "Image change detection algorithms: A systematic survey," IEEE Transactions on Image Processing, vol. 14, no. 3, pp. 294-306, Mar. 2005.

[12] L. Bruzzone and D. F. Prieto, "A minimum-cost thresholding technique for unsupervised change detection," International Journal of Remote Sensing, vol. 21, no. 18, pp. 3539-3544, Dec. 2000.

[13] F. Tung and E. LeDrew, "The determination of optimal threshold levels for change detection using various accuracy indexes," Photogrammetric Engineering and Remote Sensing, vol. 54, no. 10, pp. 1449-1454, Oct. 1988.

[14] R. S. Lunetta, J. F. Knight, J. Ediriwickrema, J. G. Lyon, and L. D. Worthy, "Land-cover change detection using multi-temporal MODIS NDVI data," Remote Sensing of Environment, vol. 105, no. 2, pp. 142154, Nov. 2006

[15] R. S. Lunetta, J. Ediriwickrema, D. M. Johnson, J. Lyon, and A. Mckerrow, "Impacts of vegetation dynamics on the identification of land-cover change in a biologically complex community in North Carolina, USA," Remote Sensing of Environment, vol. 82, no. 2-3, pp. 258-270, Oct. 2002.

[16] R. S. Lunetta et al., "NALC/Mexico land-cover mapping results: implications for assessing landscape condition," International Journal of Remote Sensing, vol. 23, no. 16, pp. 3129-3148, Aug. 2002.

[17] J. Borak, E. Lambin, and A. Strahler, "The use of temporal metrics for land cover change detection at coarse spatial scales," International Journal of Remote Sensing, vol. 21, no. 6, pp. 1415-1432, Apr. 2000.

[18] W. Kleynhans et al., "Improving land cover class separation using an extended Kalman filter on MODIS NDVI time series data," IEEE Geoscience and Remote Sensing Letters, vol. 7, no. 2, pp. 381-385, Apr. 2010

[19] Y. Li, J. Chen, R. Lu, P. Gong, and T. Yue, "Study on land cover change detection method based on NDVI time series batasets: Change detection indexes design," in IEEE International Geoscience and Remote Sensing Symposium, vol. 1-8, Seoul, South Korea, July 2005, pp. 2323-2326.

[20] D. G. Brown et al., "Stochastic simulation of land-cover change using geostatistics and generalized additive models," Photogrammetric Engineering and Remote Sensing, vol. 68, no. 10, pp. 1051-1061, Oct. 2002.

[21] C. Schaaf et al., "First operational BRDF, albedo nadir reflectance products from MODIS," Remote Sensing of Environment, vol. 83, no. 1/2, pp. $135-148$, Nov. 2002.

[22] D. Lu and Q. Weng, "A survey of image classification methods and techniques for improving classification performance," International Journal of Remote Sensing, vol. 28, no. 5, pp. 823-870, Jan. 2007.

[23] A. Strahler, "MODIS land cover product algorithm theoretical basis document (ATBD)," Boston University, Boston, MA, 1999. [Online] Available: Center for Remote Sensing

[24] C. Justice et al., "The MODIS fire products," Remote Sensing of Environment, vol. 83, no. 1-2, pp. 244-262, Nov. 2002. 